\title{
Tungstate as a transition state analog for catalysis by alkaline phosphatase
}

Ariana Peck ${ }^{1}$, Fanny Sunden ${ }^{1}$, Logan D. Andrews ${ }^{2,3}$, Vijay S. Pande ${ }^{4}$, Daniel Herschlag ${ }^{1,4}$

${ }^{1}$ Department of Biochemistry, Stanford University, Beckman Center B400, Stanford, California, 94305, United States

${ }^{2}$ Department of Chemical and Systems Biology, Stanford University, Beckman Center B400, Stanford, California, 94305, United States

${ }^{3}$ Permanent address: Achaogen, South San Francisco, California, 94080, United States

${ }^{4}$ Department of Chemistry, Stanford University, Beckman Center B400, Stanford, California, 94305, United States

Correspondence to Daniel Herschlag: herschla@stanford.edu 


\begin{abstract}
The catalytic mechanisms underlying E. coli alkaline phosphatase's (AP) remarkable rate enhancement have been probed extensively. Past work indicated that whereas the serine nucleophile (Ser102) electrostatically repels the product phosphate, another oxyanion, tungstate, binds more strongly in the presence of Ser102. These results predict a covalent bond between the serine nucleophile and tungstate, a model that we test herein. The crystal structure of tungstate-bound alkaline phosphatase provides evidence for a covalent adduct model and further shows that the ligand adopts trigonal bipyramidal geometry, which is infrequently observed for tungstate in small molecules and other active sites but mirrors the geometry of the presumed phosphoryl transfer transition state. The AP active site is known to stabilize another oxyanion, vanadate, in trigonal bipyramidal geometry, but the extent to which binding of either ligand reproduces the energetics of the transition state cannot be deduced from structural inspection alone. To test for transition state analog behavior, we determined the relationship between catalytic activity and affinity for tungstate and vanadate for a series of nineteen AP mutants. Affinity and activity were highly correlated for tungstate $\left(r^{2}=0.89\right)$ but not vanadate $\left(r^{2}=0.23\right)$, indicating that the tungstate AP complex may better mimic this enzyme's transition state properties. The results herein suggest that tungstate will be a valuable tool for further dissecting AP catalysis and may prove helpful in mechanistic studies of other phosphoryl transfer enzymes.
\end{abstract}




\section{Introduction}

E. coli alkaline phosphatase (AP) is a powerful model system for studying general mechanisms by which enzymes accelerate chemical reactions [1,2]. As a nonspecific phosphatase, AP faces the challenge of hydrolyzing diverse phosphate monoesters by interacting exclusively with the transferred phosphoryl group and the leaving group oxygen atom but no other atoms of the leaving group during catalysis (Fig. 1a, b). Despite this, the rate enhancement conferred by AP is up to $10^{27}$-fold, one of the largest recorded for enzymes [2]. The AP active site consists of a bimetallic $\mathrm{Zn}^{2+}$ core that activates the proximal Ser102 nucleophile and stabilizes charge that develops on the leaving group oxygen atom. Additional stabilization of the phosphoryl transfer transition state is thought to be provided by Arg166 and active site water molecules coordinated to the $\mathrm{Mg}^{2+}$ ion and hydrogen bonded to Lys328 (Fig. 1b) [3-5]. The tetrahedral geometry of the substrate is thought to achieve trigonal bipyramidal geometry in the phosphoryl transfer transition state [1].

Prior mechanistic work that probed AP's affinity for differentially protonated phosphate species used tungstate as a control since these anions have different $\mathrm{p} K_{\mathrm{a}}$ values. An intriguing observation from this study was that the binding affinity of the two ligands changes distinctly with mutation: specifically, while ablation of active site Arg166 reduces affinity for both anions at $\mathrm{pH} \mathrm{8,} \mathrm{additional} \mathrm{removal} \mathrm{of} \mathrm{the} \mathrm{Ser102} \mathrm{nucleophile} \mathrm{increases} \mathrm{phosphate} \mathrm{affinity}$ by 1200 -fold while reducing tungstate affinity by 5-fold (Fig. 1c) [6]. Stronger tungstate binding in the presence of Ser102 could arise from formation of a covalent adduct between the serine nucleophile and tungstate, a model that we test herein. Because tungstate in other active sites most frequently adopts tetrahedral [7-14] and more rarely trigonal bipyramidal [7,15] or octahedral geometries [16,17] (Table S1), detailed structural inspection of AP in complex with tungstate would additionally provide insight into the extent to which the AP active site stabilizes tungstate in a geometry that matches the presumed trigonal bipyramidal geometry of the transition state during phosphomonoester hydrolysis (Fig. 1b). Nevertheless, structural mimicry of the presumed transition state geometry does not guarantee that a ligand reproduces the energetics of transition state binding. We have therefore investigated both the structural and energetic properties of tungstate binding to the AP active site, and compared tungstate binding to that of vanadate, a putative transition state analog, and phosphate, a known ground state mimic.

\section{Results and Discussion}

To test the model that Ser102 forms a covalent adduct with tungstate, a crystal structure of tungstate-bound AP was obtained at $2.0 \AA$ resolution (Table 1). AP is a dimer, and the presence of a ligand in the active site of each monomer was evident from positive density in the difference map that appeared when the structure was modeled as the apo enzyme. We took advantage of density peaks in an anomalous scattering map generated from the same dataset to confirm that the ligand was not phosphate (Fig. S1a), which does not have an absorption edge near the wavelength used for data collection. The anomalous scattering map also confirmed that the $\mathrm{Mg}^{2+}$ site was occupied by a $\mathrm{Zn}^{2+}$ ion, as residual density in the difference map suggested (Fig. S1a, b). Whereas prior work has demonstrated that the metal content of AP in solution is two $\mathrm{Zn}^{2+}$ ions and one $\mathrm{Mg}^{2+}$ ion and per active site [4,18], substitution at the $\mathrm{Mg}^{2+}$ site has previously been observed crystallographically [19]. Comparison of two previously obtained sub-2 
resolution structures of phosphate-bound $\mathrm{AP}$, one with full $\mathrm{Mg}^{2+}$ occupancy (3TG0 from ref. [20]) and the other with partial $\mathrm{Zn}^{2+}$ occupancy at the third metal site (1ED8 from ref. [20]), suggests that $\mathrm{Zn}^{2+}$ substitution does not perturb the positions, within the coordinate error of the models, of the water molecules coordinated by the metal ion at this site. Thus, this substitution at the third metal site is not expected to affect interpretation of the structural data presented herein.

The tungstate molecule of our new structure was modeled in stages to most precisely position the tungstate atoms and avoid model bias. The anomalous scattering map was used to locate the tungsten atom relative to the adjacent $\mathrm{Zn}^{2+}$ ions (Fig. S1b). Refining the model with a tungsten atom in the active site left residual density peaks in the difference map that corresponded to the oxygen atoms of the tungstate (Fig. S1c); three of these peaks were roughly coplanar with the tungsten atom and another peak was approximately colinear with the tungsten atom and Ser102 oxygen atom, consistent with trigonal bipyramidal geometry. These difference map peaks were absent in the final model that contained a partially occupied tungstate molecule covalently bound to Ser102 and in a trigonal bipyramidal configuration (Fig. S1d). As a further test of this geometry, a simulated annealing omit map was calculated in which the ligand oxygen atoms were omitted. Again, three density peaks coplanar with and spaced $\sim 120^{\circ}$ around the tungsten atom and a fourth peak colinear with the tungsten and Ser102 oxygen atom were observed, consistent with the modeled ligand geometry (Fig. 2a).

The structure of tungstate-bound AP supports the prediction that tungstate forms a covalent adduct with the serine nucleophile. The $\mathrm{W}-\mathrm{O}_{\text {nuc }}$ distance is $2.2 \AA$, similar to the average distance of $2.1 \AA$ between the non-nucleophile oxygen atoms and tungsten atom. (The coordinate error for this structure is $0.16 \AA$; see Methods). The sum of the ionic radii of tungsten and oxygen yields an expected distance of $1.86 \AA$ for a covalent W-O bond [21]; however this neglects partial double bond character and assumes that the tungsten atom is in its maximal and preferred +6 oxidation state. These effects are expected to reduce and increase the covalent W-O bond distance, respectively, and this distance may be further perturbed by ligand geometry and additional bonds to the oxygen ligands [21,22]. Indeed, W-O bond lengths span 1.66 to $2.18 \AA$ in small molecule crystal structures of pentacoordinate tungstate (WO 5$)$, with mean distances of 1.70 and $1.90 \AA$ when the oxygen atom has no other covalent bond or is esterified, respectively (Table S2, Fig. S2). The W-O bonds observed in the tungstate $\bullet$ AP crystal structure are uniformly longer than these mean values. This difference may arise because longer $\mathrm{W}-\mathrm{O}$ bonds optimize interactions between tungstate and the active site, or because differences in the properties, such as unit cell compactness and elemental composition, between small molecule and protein crystals bias the distributions of W-O bonds observed. The observation of a covalent bond between Ser102 and tungstate accounts for the favorable contribution of the serine nucleophile to tungstate affinity observed by Andrews et al. [6].

In addition to testing the model of a covalent bond with Ser102, the structure of tungstate-bound AP revealed that the AP active site stabilizes tungstate in the trigonal bipyramidal configuration of the presumed transition state. This geometry is similar to the ligand geometry observed in the $1.9 \AA$ resolution crystal structure of vanadate-bound AP 
(PDB 1B8J [23]), although tungstate may be slightly more distorted from pure trigonal bipyramidal as indicated by the greater deviation from colinearity in the O-W-O angle for the apical oxygen atoms ( $164^{\circ}$ for tungstate compared to $170^{\circ}$ for vanadate) (Fig. 2b, Table S3; see Fig. S3 for separate depictions of the phosphate-bound structures and the vanadate- and tungstate-bound structures). Similar to vanadate and non-covalently bound phosphate, tungstate appears to form two hydrogen bonds to the well-positioned side chain of Arg166 (Fig. 2b, Table S4) [20,23]. However, the distortion of the apical oxygen atoms from colinearity combined with the longer $\mathrm{W}-\mathrm{O}$ bonds appears to enable tungstate to provide superior coordination of the $\mathrm{Zn}^{2+}$ ions. All four of its $\mathrm{Zn}^{2+}-\mathrm{O}$ distances fall in the expected range of 1.95-2.14 $\AA$ [24], whereas one of these bonds is considerably longer for vanadate, non-covalent phosphate, and covalently bound phosphate $(2.4,2.7$, and $2.6 \AA$, respectively), and another is absent from covalently bound phosphate (Fig. 2b, Table S5) [20,23,25].

Unlike phosphate, tungstate and vanadate can undergo valency expansion to accommodate trigonal bipyramidal geometry. However, pentacoordinate tungstate infrequently adopts this geometry in small molecule compounds and the active sites of other phosphatases (Tables S1 and S2), so the observation of trigonal bipyramidal geometry in the AP active site would not be expected based solely on structural precedent [26]. A survey of tungsten-containing small molecules in the Cambridge Structural Database [27] reveals that tungsten coordinated exclusively by oxygen atoms is most commonly hexacoordinate with octahedral geometry (Table S2). Pentacoordinate tungsten favors square pyramidal geometry, and there are no observed instances of trigonal bipyramidal geometry with five oxygen ligands. When structures of pentacoordinate tungsten containing one non-oxygen ligand are considered, only one example of trigonal bipyramidal geometry is found, with the majority of the $41 \mathrm{WO}_{4} \mathrm{X}$ compounds favoring square pyramidal geometry (Table S2). A survey of active sites containing tungstate in the Protein Data Bank reveals a mix of tetrahedral, trigonal bipyramidal, and (in the case of histidine nucleophiles) octahedral geometries. However, with the exception of hexose phosphate phosphatase, cases that exhibit a trigonal bipyramidal configuration consistently deviate from the ideal geometry (in which the equatorial oxygen atoms are coplanar and spaced $120^{\circ}$ apart) and, unlike AP, appear to be tetra- rather than pentacoordinate due to the distance between the tungsten atom and the enzyme's nucleophile (Table S1).

The observation that wild-type AP stabilizes both tungstate and vanadate in the trigonal bipyramidal geometry of the presumed transition state prompted us to assess both oxyanions as transition state analogs. Strong binding affinity of a ligand-greater than that for substrates and products-is often considered an indication that a ligand is a transition state analog $[28,29]$. However, high binding affinity can also arise from fortuitous interactions that do not mimic transition state binding [30]. Conversely, weak binding affinity does not necessarily indicate a complete absence of transition state character in the ligand's interaction with the active site. A more stringent criterion for a transition state analog is a linear $\log -\log$ relationship between affinity and catalysis with a slope of one across a series of systematic perturbations to the enzyme (or ligand) [29,31]. High correlation in this relationship indicates that the energetics of ligand binding and transition state stabilization are sensitive to the same perturbations, and a slope of one suggests that these energetic effects are of the same magnitude for a given perturbation [29]. 
We therefore determined the relationship between ligand association constants, $K_{\mathrm{a}}$, and $k_{\text {cat }} / K_{\mathrm{M}}$ for twenty AP variants that collectively span nine orders of magnitude in reaction rate (Table 2). Binding affinities for phosphate, a ground state mimic, span only four orders of magnitude, so, despite some correlation $\left(\mathrm{r}^{2}=0.55\right)$, the correlation line's slope is substantially greater than one $(\mathrm{m}=3.3$, RMSD $=0.54$; Fig. 3a and Table 3). By contrast, the logarithms of tungstate association constants and $k_{\mathrm{cat}} / K_{\mathrm{M}}$ for the same set of mutants are highly linearly correlated $\left(\mathrm{r}^{2}\right.$ $=0.89)$ with a slope close to one $(\mathrm{m}=1.4$, RMSD $=0.28$; Fig. $3 \mathrm{~b}$ and Table 3$)$. This behavior supports tungstate as a good transition state analog, and the slope of 1.4 rather than 1 suggests that there are differences between tungstate binding and transition state stabilization that remain to be understood. Surprisingly, the log-log relationship of vanadate binding affinities and catalytic efficiencies does not show a similar linear correlation, with a slope of 3.8 and a coefficient of determination of only 0.23 (Fig. 3c and Table 3). Intriguingly, if the E322Y mutants that lack the $\mathrm{Mg}^{2+}$ ion are excluded from the fit, a strong correlation with a slope closer to one emerges (Fig. 3d and Table 3; $\left.\mathrm{r}^{2}=0.91, \mathrm{~m}=1.5, \mathrm{RMSD}=0.23\right)$. The same trends are observed when a linear model with the slope constrained to 1 is fit to each dataset (Fig. 3, red lines): phosphate and vanadate behaviors are poorly fit by this model (RMSD of 1.15 and 1.04, respectively; Fig. 3a, c and Table 3), whereas the tungstate data fit well (RMSD = 0.41, Fig. 3b and Table 3) and the vanadate data fit well but only after the E322Y-containing mutants were excluded $(\mathrm{RMSD}=0.40$, Fig. 3d and Table 3).

These observations raise the following questions: why is tungstate a superior transition state analog compared to vanadate, and why is vanadate binding affinity insensitive to loss of the $\mathrm{Mg}^{2+}$ ion? Prior work dissecting the AP active site has shown that apart from the Ser102 nucleophile and bimetallic $\mathrm{Zn}^{2+}$ core, the $\mathrm{Mg}^{2+}$ ion and $\mathrm{Arg} 166$ are the active site moieties that have the largest effects on catalysis (Table 2) [4,5]. However, the $\mathrm{Mg}^{2+}$ ion and its associated water network provide significantly more transition state specific stabilization compared to Arg166, which stabilizes both the transition and ground states (Fig. 1b) $[4,5,18]$. The relative contributions of the $\mathrm{Mg}^{2+}$ ion to binding affinity versus rate enhancement would thus be expected to probe how well a ligand mimics the energetics specific to AP's transition state, so the insensitivity of vanadate but not tungstate binding affinity to loss of the $\mathrm{Mg}^{2+}$ ion is particularly striking in the context of transition state analog behavior (Fig. 3, Tables 2 and 3).

Inherent differences in the charge distribution, protonation states, and/or favored bond lengths and geometries of vanadate and tungstate oxyanions may account for the distinct energetic contributions of the $\mathrm{Mg}^{2+}$ ion to their stabilities in the AP active site. However, our functional data suggest that the first two models do not account for the observed $\mathrm{Mg}^{2+}$ effect. Specifically, a model that attributes vanadate's and tungstate's distinct energetic properties to their different charge distributions would not predict the ligands' similar responses to ablation of Arg166 and its electrostatic interactions (Fig. 3, Table 2). Likewise, the same trends and correlations are observed across pH, providing evidence against complications from differential protonation states of the ligands (see Methods). In contrast, comparison of the AP•tungstate and AP•vanadate structures suggests that energetic differences may arise from distinct interactions between the ligand oxygen atoms and the $\mathrm{Zn}^{2+}$ core due to the longer $\mathrm{W}-\mathrm{O}$ than $\mathrm{V}-\mathrm{O}$ bond. 
Specifically, both ligands appear to interact with the $\mathrm{Mg}^{2+}$-associated water network (Fig. S4), but tungstate coordinates the $\mathrm{Zn}^{2+}(1)$ ion that presumably stabilizes the leaving group oxygen of the transition state whereas vanadate does not (Fig. 2b, Table 2). It is possible, given vanadate's bond lengths, that interactions with the $\mathrm{Mg}^{2+}$ water network and $\mathrm{Zn}^{2+}(1)$ cannot be simultaneously optimized. If this were the case, loss of the $\mathrm{Mg}^{2+}$-water network interactions might be energetically compensated by formation of a $\mathrm{Zn}^{2+}(1)$-oxygen interaction in the vanadate complex. This interaction is already present in the wild-type AP•tungstate complex (Fig. 2b, Table S5), so this complex would not experience compensation for the energetic cost of $\mathrm{Mg}^{2+}$ ion removal and could thus mimic transition state energetics. It is also possible that loss of the $\mathrm{Mg}^{2+}$ ion increases the conformational mobility of the tungstate oxygen atom that interacts with the $\mathrm{Mg}^{2+}$-associated water molecule, and this in turn destabilizes the $\mathrm{Zn}^{2+}(1)$-oxygen interaction that is present in tungstate but not vanadate. These models make distinct predictions for the properties of mutant AP complexes with tungstate and vanadate.

The finding that tungstate is covalently bound to Ser102 resolves the question of why, in contrast to phosphate, the presence of the serine nucleophile contributes favorably to tungstate affinity (Fig. 1c) [6]. The observation that the AP active site stabilizes tungstate in trigonal bipyramidal geometry is striking given how infrequently tungstate adopts this geometry in other enzyme active sites and small molecule compounds. We cannot experimentally measure the energetic cost of distortion of tungstate from the tetrahedral geometry favored in solution to the trigonal bipyramidal geometry stabilized by the AP active site. Nevertheless, this energetic penalty may account for a surprising observation: ablation of the nucleophile (from AP Arg166Ser to Ser102Gly/Arg166Ser), which results in loss of a covalent bond, only causes a 5-fold reduction in affinity (Fig. 1c) [6]. It is thus possible that the enthalpic contribution from forming the covalent bond with Ser102 is nearly fully offset by distorting tungstate to an energetically unfavorable geometry.

Stabilization of the trigonal bipyramidal geometry of the presumed transition state is observed for both tungstate and vanadate in the AP active [23]. However, tungstate, but not vanadate, passes the litmus test for a transition state analog -i.e., a linear correlation with a slope of one between the logarithms of catalytic efficiency and ligand binding affinity (Fig. 3) [32,33]. Two prior studies challenged vanadate as a transition state analog for phosphoryl transfer [33,34], and our work extends those reports in a critical way. Vanadate and tungstate have frequently been inferred to be transition state analogs based on high binding affinity to the wild-type enzyme (e.g. refs. [35-37]) or, especially for vanadate, active site stabilization of the ligand in trigonal bipyramidal geometry (reviewed in ref. [26]). To our knowledge, only two studies have performed a mutational test of vanadate's transition state analog energetics (and none have similarly examined tungstate); three and five active site mutants of RNase E [33] and protein tyrosine phosphatase [34], respectively, gave vanadate binding behavior inconsistent with the prediction of linearity for a transition state analog. Extending this analysis to nineteen mutants of AP enables us to localize vanadate's deviation from transition state analog behavior to a specific moiety -the $\mathrm{Mg}^{2+}$ ion- in the AP active site, a mechanistic insight afforded by the scope of the mutants presented herein. With this test, we further established that tungstate is a more suitable transition state analog. Studies of AP•tungstate complexes may help elucidate the 
catalytic contributions of the $\mathrm{Mg}^{2+}$ ion in AP and may be useful in future mechanistic studies of $\mathrm{AP}$ and other phosphoryl transfer enzymes.

\section{Methods}

Protein expression and purification. AP was purified from a fusion construct that contained an $\mathrm{N}$-terminal maltose binding protein tag and a C-terminal factor Xa cleavage site and StrepII tag. E. coli SM547(DE3) cells were transformed with this construct and grown at $37^{\circ} \mathrm{C}$ in rich media supplemented with glucose $(10 \mathrm{~g}$ of tryptone, $5 \mathrm{~g}$ of yeast extract, $5 \mathrm{~g}$ of $\mathrm{NaCl}$, and $2 \mathrm{~g}$ of glucose per liter). Once the OD600 reached 0.6, expression of the AP fusion construct was induced by addition of IPTG to $0.3 \mathrm{mM}$. Cultures were grown for an additional $16-20 \mathrm{~h}$ at $30^{\circ} \mathrm{C}$. Cells were harvested by centrifugation at $4400 \times \mathrm{g}$ for $20 \mathrm{~min}$ and lysed by osmotic shock. The pellet was resuspended in $800 \mathrm{~mL} 20 \%$ sucrose solution (30 mM Tris- $\mathrm{HCl}, \mathrm{pH} 8.0,1 \mathrm{mM}$ EDTA) per original $2 \mathrm{~L}$ culture and incubated at $4^{\circ} \mathrm{C}$ for $10 \mathrm{~min}$ on a shaking table. Cells were re-pelleted by centrifugation at 13,000×g for $10 \mathrm{~min}$ and resuspended in column buffer ( $10 \mathrm{mM}$ Tris- $\mathrm{HCl}, \mathrm{pH} 7.4,200 \mathrm{mM} \mathrm{NaCl}$, and $10 \mu \mathrm{M} \mathrm{ZnCl}_{2}$ ) at $4^{\circ} \mathrm{C}$. The sample was passed over a $10 \mathrm{~mL}$ amylose resin gravity column (New England BioLabs, Ipswich, MA). The column was washed with 10 column volumes of column buffer and eluted with the same buffer supplemented with $10 \mathrm{mM}$ maltose. Fractions containing protein were concentrated by centrifugation through a filter (Amicon) with a $10 \mathrm{kDa}$ cut-off and bufferexchanged twice into $10 \mathrm{mM}$ Na-MOPS, pH 7.0, $50 \mathrm{mM} \mathrm{NaCl}, 100 \mu \mathrm{M} \mathrm{ZnCl}_{2}$, and $1.0 \mathrm{mM} \mathrm{MgCl}_{2}$. In the case of $\mathrm{AP}$ mutants containing he $\mathrm{E} 322 \mathrm{Y}$ mutation, $\mathrm{MgCl}_{2}$ was omitted from the buffer. Purity was determined to be greater than $95 \%$ by SDS-PAGE gel electrophoresis with staining by Coomassie Blue.

Inhibition measurements. Inhibition constants for phosphate, tungstate, and vanadate were measured in $0.1 \mathrm{M}$ MOPS, pH 8.0, $0.5 \mathrm{M} \mathrm{NaCl}, 100 \mu \mathrm{M} \mathrm{ZnCl}_{2}$, and $500 \mu \mathrm{M} \mathrm{MgCl}_{2}$ at $25^{\circ} \mathrm{C}$, except for $\mathrm{E} 322 \mathrm{Y}$ mutants, for which $\mathrm{MgCl}_{2}$ was excluded. The maltose binding protein tag was not excised from AP for these measurements; this tag has previously been shown to not affect AP's rate constant [4,38]. Inhibitor concentration was varied at least 5-fold below and above the observed $K_{\mathrm{i}}$ at a constant concentration of enzyme and a sub-saturating concentration of methyl $p$-nitrophenol phosphate, a diester substrate. To prevent polymerization of vanadate and tungstate, stock ligand was stored at $\mathrm{pH} 13$, and maximum concentrations of 4.6 and $13 \mathrm{mM}$, respectively, of the inhibitor were used. Formation of the product $p$-nitrophenolate was monitored continuously at $400 \mathrm{~nm}$. The inhibition constant was calculated with a nonlinear least-squares fit to the data using the following equation for competitive inhibition:

$$
k_{c a t} / K_{M, a p p}=\frac{k_{c a t} / K_{M, o b s}}{1+\frac{[I]}{K_{i}}}
$$

The above equation is most commonly used to describe non-covalent inhibition, but is also applicable to covalent inhibition when the non-covalent and covalently bound species equilibrate more quickly than the timescale of the measurement. The measured rates were linear over time at all concentrations of inhibitor, indicating an absence of time-dependence to the inhibition and thus fast equilibration of the covalent and non-covalent species relative to the measurement.

Correlation between $\log \left(k_{\mathrm{cat}} / K_{\mathrm{M}}\right)$ and $\log \left(K_{\mathrm{a}}\right)$ was modeled by total least-squares regression. The slope for the linear fit was calculated by performing principal component analysis; the ratio of the values of the first eigenvector of the 
covariance matrix yields the slope, $\mathrm{m}$, and the $\mathrm{y}$-intercept, $\mathrm{b}$, can be recovered from: $\mathrm{b}=\bar{y}-\mathrm{m} \bar{x}$. Additionally, the optimal linear fit was calculated when the slope was constrained to $\mathrm{m}=1$. Goodness of fit was assessed by computing the root mean square deviation (RMSD) of the minimal (orthogonal) distance between each data point $\left(\log \left(K_{\mathrm{a}}\right), \log \left(k_{\mathrm{cat}} / K_{\mathrm{M}}\right)\right)$ and each linear model. Finally, the coefficient of determination, $\mathrm{r}^{2}$, was computed from the square of the Pearson correlation coefficient; the latter is given by the following formula:

$$
r=\frac{\sum_{i=1}^{n}\left(x_{i}-\bar{x}\right)\left(y_{i}-\bar{y}\right)}{\sqrt{\sum_{i=1}^{n}\left(x_{i}-\bar{x}\right)^{2}} \sqrt{\sum_{i=1}^{n}\left(y_{i}-\bar{y}\right)^{2}}}
$$

Inhibition constants were additionally measured at $\mathrm{pH} 9.0$ for 16 of the 19 mutants listed in Table 2, and the same trends and correlations as reported in Fig. 3 were observed (data not shown). We were unable to measure inhibition constants below $\mathrm{pH} 8.0$ due to polymerization of vanadate and tungstate at neutral $\mathrm{pH}$ and above $\mathrm{pH} 9.0$ due to an enzymatic $\mathrm{pK}_{\mathrm{a}}$ [6] that raises the inhibition constant above the threshold that we can measure for most mutants.

Crystallization. WT AP was purified as described above, and the maltose binding protein tag was cleaved with factor Xa. Enzyme and tag were separated by passage over a $5 \mathrm{~mL}$ HiTrap Q HP column (GE Healthcare, Amersham, UK). Enzyme was buffer-exchanged into $10 \mathrm{mM} \mathrm{Na-MOPS,} \mathrm{pH} \mathrm{8.0,50} \mathrm{mM} \mathrm{NaCl,} 10 \mu \mathrm{M} \mathrm{ZnCl}_{2}$, and $100 \mu \mathrm{M} \mathrm{MgCl}_{2}$, concentrated to $200 \mu \mathrm{M}$, and pre-incubated with $500 \mu \mathrm{M}$ sodium tungstate. Crystallization was performed by the hanging drop method at $18^{\circ} \mathrm{C}$, with equal volumes $(2 \mu \mathrm{L}$ each) of enzyme and precipitant solution (18\% PEG3350, 0.2 M ammonium fluoride) placed over a reservoir of $1 \mathrm{~mL}$ precipitant solution. Crystals were soaked in cryoprotectant solution (30\% glycerol, $0.1 \mathrm{mM}$ Bis-Tris, $\mathrm{pH} 5.0$, and $0.2 \mathrm{mM}$ ammonium sulfate) and frozen in liquid nitrogen. Crystallographic data were collected at Stanford Linear Accelerator beamline 12-2 at a wavelength of $0.9795 \AA$.

Structure determination. Data integration was performed with MOSFLM [39]; scaling and merging were carried out in AIMLESS [40]. Five percent of reflections were set aside to calculate $R_{\text {free }}$. Molecular replacement was completed with PHASER [41] using WT AP (PDB 3TG0 [20]) stripped of ligand and metals as a search model. Alternating rounds of manual model building and automated refinement were performed with COOT [42] and REFMAC5 [43], respectively. The tungstate ion was modeled in stages near the end of the refinement process. First, an anomalous scattering map was generated from the scaled dataset and partially-refined model using PHENIX [44], which enabled positioning of the tungsten atom into the center of the anomalous scattering peak. After a round of automated refinement, negative density appeared in the difference map at the position of the tungsten atom, so its occupancy was reduced to 0.5. A tetrahedral tungstate ion was then loaded from the JLigand library [45] and substituted for the tungsten atom. Automated refinement corrected the geometry to trigonal bipyramidal, consistent with the appearance of the difference density map prior to addition of the tungstate oxygen atoms. Omit maps generated in PHENIX [44] provided additional validation of the trigonal bipyramidal geometry. Two positive density peaks remained in the active site after modeling in the tungstate; these were modeled as half-occupied water molecules that coordinate the $\mathrm{Zn}^{2+}$ ions. Stereochemistry was validated by MOLPROBITY [46], and images were generated with PYMOL [47]. Coordinate error was determined by the diffraction-data precision indicator (DPI) estimate [48]. 
Keywords: enzymes, phosphoryl transfer, transition state, active sites

Abbreviations used: AP, alkaline phosphatase; $\mathrm{O}_{\text {nuc }}$, nucleophilic oxygen atom; $\mathrm{K}_{\mathrm{a}}$, association constant

\section{Accession numbers}

The accession number for the tungstate $\bullet$ AP crystal structure is PDB ID: $\mathbf{5 C 6 6}$.

\section{Acknowledgments}

This work was funded by a grant from the US National Institutes of Health to DH (GM49243). A.P. was funded by a National Science Foundation Graduate Research Fellowship. L.D.A was supported in part by an NIH training grant (R1GM064798). The SSRL Structural Molecular Biology Program receives support from the Department of Energy, Office of Biological and Environmental Research, the National Institutes of Health, National Center for Research Resources, Biomedical Technology Program, and the National Institute of General Medical Sciences. We thank Daniel Roston and Qiang Cui for instructive discussions, Susanne Ressl for help with crystallographic data collection, Artem Lyubimov and James Fraser for useful discussions about crystallographic refinement, Ishraq AlSadhan for help with enzyme purification, and Julia Salzman for advice about statistics. We are grateful to members of the Herschlag group for discussions and comments on the manuscript.

\section{References}

[1] Coleman JE. Structure and mechanism of alkaline phosphatase. Annu Rev Biophys Biomol Struct 1992;21:441-83. doi:10.1146/annurev.bb.21.060192.002301.

[2] Lassila JK, Zalatan JG, Herschlag D. Biological phosphoryl-transfer reactions: understanding mechanism and catalysis. Annu Rev Biochem 2011;80:669-702. doi:10.1146/annurev-biochem-060409-092741.

[3] O'Brien PJ, Herschlag D. Alkaline Phosphatase Revisited: Hydrolysis of Alkyl Phosphates $\dagger$. Biochemistry 2002;41:3207-25. doi:10.1021/bi012166y.

[4] Zalatan JG, Fenn TD, Herschlag D. Comparative enzymology in the alkaline phosphatase superfamily to determine the catalytic role of an active-site metal ion. J Mol Biol 2008;384:1174-89.

doi:10.1016/j.jmb.2008.09.059.

[5] O’Brien PJ, Lassila JK, Fenn TD, Zalatan JG, Herschlag D. Arginine coordination in enzymatic phosphoryl transfer: evaluation of the effect of Arg 166 mutations in Escherichia coli alkaline phosphatase. Biochemistry 2008;47:7663-72. doi:10.1021/bi800545n.

[6] Andrews LD, Fenn TD, Herschlag D. Ground State Destabilization by Anionic Nucleophiles Contributes to the Activity of Phosphoryl Transfer Enzymes. PLoS Biol 2013;11. doi:10.1371/journal.pbio.1001599.

[7] Lu Z, Dunaway-Mariano D, Allen KN. The catalytic scaffold of the haloalkanoic acid dehalogenase enzyme superfamily acts as a mold for the trigonal bipyramidal transition state. Proc Natl Acad Sci U S A 2008;105:5687-92. doi:10.1073/pnas.0710800105. 
[8] Lu Z, Wang L, Dunaway-Mariano D, Allen KN. Structure-function analysis of 2-Keto-3-deoxy-D-glyceroD-galactonononate- 9-phosphate Phosphatase defines specificity elements in type C0 haloalkanoate dehalogenase family members. J Biol Chem 2009;284:1224-33. doi:10.1074/jbc.M807056200.

[9] Peisach E, Selengut JD, Dunaway-Mariano D, Allen KN. X-ray crystal structure of the hypothetical phosphotyrosine phosphatase MDP-1 of the haloacid dehalogenase superfamily. Biochemistry 2004;43:12770-9. doi:10.1021/bi0490688.

[10] Chu HM, Wang AHJ. Enzyme-substrate interactions revealed by the crystal structures of the archaeal Sulfolobus PTP-fold phosphatase and its phosphopeptide complexes. Proteins Struct Funct Genet 2007;66:996-1003. doi:10.1002/prot.21262.

[11] Brandão TAS, Robinson H, Johnson SJ, Hengge AC. impaired acid catalysis by mutation of a protein loop hinge residue in a YopH mutant revealed by crystal structures. J Am Chem Soc 2009;131:778-86. doi:10.1021/ja807418b.

[12] Reynolds RA, Yem AW, Wolfe CL, Deibel MR, Chidester CG, Watenpaugh KD. Crystal structure of the catalytic subunit of Cdc25B required for G2/M phase transition of the cell cycle. J Mol Biol 1999;293:55968. doi:10.1006/jmbi.1999.3168.

[13] Hamda K, Kato M, Shimizu T, Ihara K, Mizuno T, Hakoshima T. Crystal structure of the protein histidine phosphatase SixA in the multistep His-Asp phosphorelay. Genes to Cells 2005;10:1-11. doi:10.1111/j.13652443.2005.00817.x.

[14] Chen Y, Jakoncic J, Wang J, Zheng X, Carpino N, Nassar N. Structural and functional characterization of the $\mathrm{C}$-terminal domain of the ecdysteroid phosphate phosphatase from Bombyx mori reveals a new enzymatic activity. Biochemistry 2008;47:12135-45. doi:10.1021/bi801318w.

[15] Felts RL, Ou Z, Reilly TJ, Tanner JJ. Structure of recombinant Haemophilus influenzae e (P4) acid phosphatase reveals a new member of the haloacid dehalogenase superfamily. Biochemistry 2007;46:11110-9. doi:10.1021/bi701016m.

[16] Davies DR, Interthal H, Champoux JJ, Hol WGJ. Insights into substrate binding and catalytic mechanism of human tyrosyl-DNA phosphodiesterase (Tdp1) from vanadate and tungstate-inhibited structures. J Mol Biol 2002;324:917-32. doi:10.1016/S0022-2836(02)01154-3.

[17] Leiros I, McSweeney S, Hough E. The reaction mechanism of phospholipase D from Streptomyces sp. strain PMF. Snapshots along the reaction pathway reveal a pentacoordinate reaction intermediate and an unexpected final product. J Mol Biol 2004;339:805-20. doi:10.1016/j.jmb.2004.04.003.

[18] Sunden F, Peck A, Salzman J, Ressl S, Herschlag D. Extensive site-directed mutagenesis reveals interconnected functional units in the alkaline phosphatase active site. Elife 2015;4:1-31. doi:10.7554/eLife.06181.

[19] Stec B, Holtz KM, Kantrowitz ER. A revised mechanism for the alkaline phosphatase reaction involving 
three metal ions. J Mol Biol 2000;299:1303-11. doi:10.1006/jmbi.2000.3799.

[20] Bobyr E, Lassila JK, Wiersma-Koch HI, Fenn TD, Lee JJ, Nikolic-Hughes I, et al. High-resolution analysis of $\mathrm{Zn} 2+$ coordination in the alkaline phosphatase superfamily by EXAFS and x-ray crystallography. J Mol Biol 2012;415:102-17. doi:10.1016/j.jmb.2011.10.040.

[21] Shannon RD. Revised effective ionic radii and systematic studies of interatomic distances in halides and chalcogenides. Acta Crystallogr Sect A 1976;32:751-67. doi:10.1107/S0567739476001551.

[22] Harris SE, Orpen a. G, Bruno IJ, Taylor R. Factors affecting d-block metal-ligand bond lengths: Toward an automated library of molecular geometry for metal complexes. J Chem Inf Model 2005;45:1727-48. doi:10.1021/ci0500785.

[23] Holtz KM, Stec B, Kantrowitz ER. A Model of the Transition State in the Alkaline Phosphatase Reaction. J Biol Chem 1999;274:8351-4.

[24] Harding MM. Geometry of metal-ligand interactions in proteins. Acta Crystallogr Sect D Biol Crystallogr 2001;57:401-11. doi:10.1107/S0907444900019168.

[25] Murphy JE, Stec B, Ma L, Kantrowitz ER. Trapping and visualization of a covalent enzyme-phosphate intermediate. Nat Struct Biol 1997;4:618-22. doi:10.1038/nsb0897-618.

[26] Davies DR, Hol WGJ. The power of vanadate in crystallographic investigations of phosphoryl transfer enzymes. FEBS Lett 2004;577:315-21. doi:10.1016/j.febslet.2004.10.022.

[27] Allen FH. The Cambridge Structural Database: A quarter of a million crystal structures and rising. Acta Crystallogr Sect B Struct Sci 2002;58:380-8. doi:10.1107/S0108768102003890.

[28] Wolfenden R. Transition state analogues for enzyme catalysis. Nature 1969;223:704-5. doi:10.1038/223704a0.

[29] Mader MM, Bartlett P a. Binding Energy and Catalysis: The Implications for Transition-State Analogs and Catalytic Antibodies. Chem Rev 1997;97:1281-302. doi:10.1021/cr960435y.

[30] Wolfenden R. Transition State Analog Inhibitors and Enzyme Catalysis. Annu Rev Biophys Bioeng 1976;5:271-306. doi:10.1146/annurev.bb.05.060176.001415.

[31] Messmore JM, Raines RT. Pentavalent organo-vanadates as transition state analogues for phosphoryl transfer reactions. J Am Chem Soc 2000;122:9911-6. doi:10.1021/ja0021058.

[32] Bartlett PA, Marlowe CK. Phosphonamidates as transition-state analogue inhibitors of thermolysin. Biochemistry 1983;22:4618-24. doi:10.1021/bi00289a002.

[33] Messmore JM, Raines RT. Pentavalent organo-vanadates as transition state analogues for phosphoryl transfer reactions. J Am Chem Soc 2000;122:9911-6. doi:10.1021/ja0021058. 
[34] Deng H, Callender R, Huang Z, Zhang Z-Y. Is the PTPase-vanadate complex a true transition state analogue? Biochemistry 2002;41:5865-72. doi:10.1021/bi016097z.

[35] VanEtten RL, Waymack PP, Rehkop DM. Transition metal ion inhibition of enzyme-catalyzed phosphate ester displacement reactions. J Am Chem Soc 1974;96:6782-5. doi:10.1021/ja00828a053.

[36] Georgalis Y, Zouni A, Hahn U, Saenger W. Synthesis and kinetic study of transition state analogs for ribonuclease T1. Biochim Biophys Acta 1991;1118:1-5.

[37] Stankiewicz PJ, Gresser MJ. Inhibition of phosphatase and sulfatase by transition-state analogues. Biochemistry 1988;27:206-12. doi:10.1021/bi00401a031.

[38] O’Brien PJ, Herschlag D. Functional interrelationships in the alkaline phosphatase superfamily: Phosphodiesterase activity of Escherichia coli alkaline phosphatase. Biochemistry 2001;40:5691-9. doi:10.1021/bi0028892.

[39] Battye TGG, Kontogiannis L, Johnson O, Powell HR, Leslie AGW. iMOSFLM: A new graphical interface for diffraction-image processing with MOSFLM. Acta Crystallogr Sect D Biol Crystallogr 2011;67:271-81. doi:10.1107/S0907444910048675.

[40] Evans PR, Murshudov GN. How good are my data and what is the resolution? Acta Crystallogr Sect D Biol Crystallogr 2013;69:1204-14. doi:10.1107/S0907444913000061.

[41] McCoy AJ, Grosse-Kunstleve RW, Adams PD, Winn MD, Storoni LC, Read RJ. Phaser crystallographic software. J Appl Crystallogr 2007;40:658-74. doi:10.1107/S0021889807021206.

[42] Emsley P, Lohkamp B, Scott WG, Cowtan K. Features and development of Coot. Acta Crystallogr Sect D Biol Crystallogr 2010;66:486-501. doi:10.1107/S0907444910007493.

[43] Murshudov GN, Skubák P, Lebedev AA, Pannu NS, Steiner RA, Nicholls RA, et al. REFMAC5 for the refinement of macromolecular crystal structures. Acta Crystallogr Sect D Biol Crystallogr 2011;67:355-67. doi:10.1107/S0907444911001314.

[44] Adams PD, Afonine P V., Bunkóczi G, Chen VB, Davis IW, Echols N, et al. PHENIX: A comprehensive Python-based system for macromolecular structure solution. Acta Crystallogr Sect D Biol Crystallogr 2010;66:213-21. doi:10.1107/S0907444909052925.

[45] Lebedev AA, Young P, Isupov MN, Moroz O V., Vagin AA, Murshudov GN. JLigand: A graphical tool for the CCP4 template-restraint library. Acta Crystallogr Sect D Biol Crystallogr 2012;68:431-40. doi:10.1107/S090744491200251X.

[46] Chen VB, Arendall WB, Headd JJ, Keedy DA, Immormino RM, Kapral GJ, et al. MolProbity: All-atom structure validation for macromolecular crystallography. Acta Crystallogr Sect D Biol Crystallogr 2010;66:12-21. doi:10.1107/S0907444909042073. 
[47] DeLano WL. The PyMOL Molecular Graphics System. Schrödinger LLC Wwwpymolorg 2002;Version 1.:http://www.pymol.org. doi:citeulike-article-id:240061.

[48] Murshudov G, Dodson E. Simplified error estimation a la Cruickshank in macromolecular crystallography. CCP4 Newsl 1997. 


\section{Legends}

Fig. 1: Summary of the AP active site. (a) Reaction scheme for the phosphomonoester hydrolysis catalyzed by AP. ROP and E-P represent the phosphate monoester substrate and covalent seryl-phosphate intermediate, respectively. (b) Schematic of the AP active site drawn with partial bonds as in the presumed phosphoryl transfer transition state.

(c) Inhibition constants were calculated for the $\mathrm{AP} \cdot \mathrm{HPO}_{4}{ }^{2-}$ and $\mathrm{AP} \bullet \mathrm{WO}_{4}{ }^{2-}$ complexes for select AP mutants at $\mathrm{pH} 8$. From Andrews et al. [6].

\section{Table 1: X-ray crystallographic data collection and refinement statistics}

Fig. 2: Structure of the tungstate-bound AP active site and in comparison to other bound anionic ligands. (a) Overlay of maps of the $2 \mathrm{~F}_{\mathrm{o}}-\mathrm{F}_{\mathrm{c}}$ electron density (contoured at $1 \sigma$ ) of the final model (blue) and the $\mathrm{F}_{\mathrm{o}}-\mathrm{F}_{\mathrm{c}}$ map electron density (contoured at $4 \sigma$ ) from the simulated annealing omit map in which the oxygen atoms of the tungstate ion were omitted (green). $\mathrm{Zn}^{2+}$ ions are colored dark grey; tungstate, light blue; Ser102 and Arg166, light grey; waters, red. For clarity, only one active site is shown. (b) Crystal structures of AP bound to tungstate, vanadate, and phosphate (both covalently and non-covalently) were overlaid to compare the positions of ligands with respect to Ser102, $\mathrm{Arg} 166$, the active site $\mathrm{Zn}^{2+}$ and $\mathrm{Mg}^{2+}$ ions, and the $\mathrm{Mg}^{2+}$-coordinated water molecules. In the tungstate structure, the ligand was modeled in at partial occupancy, with partially-occupied water molecules at sites that presumably coordinate the $\mathrm{Zn}^{2+}$ ions in the fraction of enzyme without bound tungstate. The PDB codes for vanadate, non-covalently-bound phosphate, and covalently-bound phosphate are 1B8J, 3TG0, and 1HJK, respectively.

\section{Table 2: Inhibition constants and catalytic efficiencies for AP mutants}

Fig. 3: Transition state analog behavior of phosphate, tungstate, and vanadate. Correlations between the $\log$ of $k_{\mathrm{ca}} / K_{\mathrm{M}}$ and log of association constant, $K_{\mathrm{a}}$, for (a) phosphate, (b) tungstate, and (c) vanadate. The linear fit from total least squares regression is shown by the grey lines, and the slope (m) and root mean square deviation (RMSD) are given in grey at the bottom right of each plot. The red lines show linear regressions with the slope constrained to 1 , and the $\mathrm{m}$ and RMSD values are given in red. The value for the coefficient of determination, $\mathrm{r}^{2}$, is given for each dataset in black. For vanadate, the linear fit was also calculated excluding mutants with the $\mathrm{Mg}^{2+}$ site ablated (d). The AP variants used are listed in (e); mutants with an intact $\mathrm{Mg}^{2+}$ site are represented by diamonds, and those deficient in $\mathrm{Mg}^{2+}$ binding due to the presence of the E322Y mutation are indicated by squares. Arrows denote mutants for which only an upper limit for $K_{\mathrm{a}}$ could be determined; these mutants were not included in the linear fits. Error bars correspond to standard deviations from 2-3 independent experiments.

\section{Table 3: Transition state analog behavior for phosphate, tungstate and vanadate}




\section{Figure 1}

a

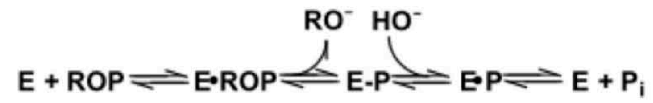

c

\begin{tabular}{ccc} 
& \multicolumn{2}{c}{$K_{\mathrm{d}}(\mu \mathrm{M})$} \\
\cline { 2 - 3 } & Phosphate & Tungstate \\
\hline WT & 0.46 & 0.57 \\
R166S & 110 & 170 \\
S102G/R166S & 0.093 & 890 \\
\hline
\end{tabular}

b

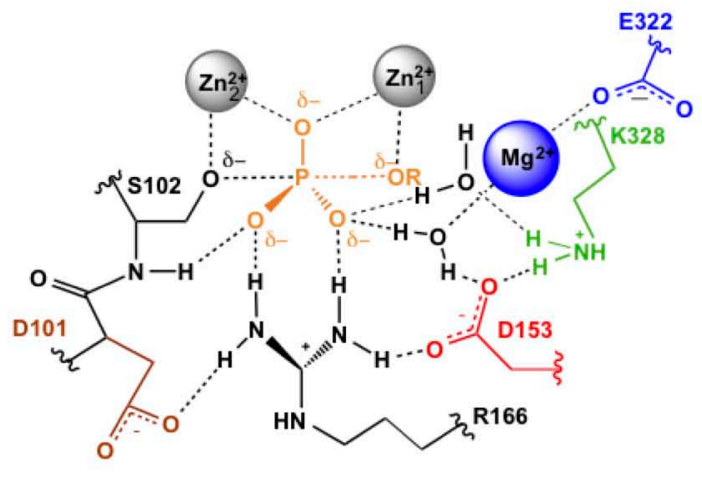


Figure 2

a

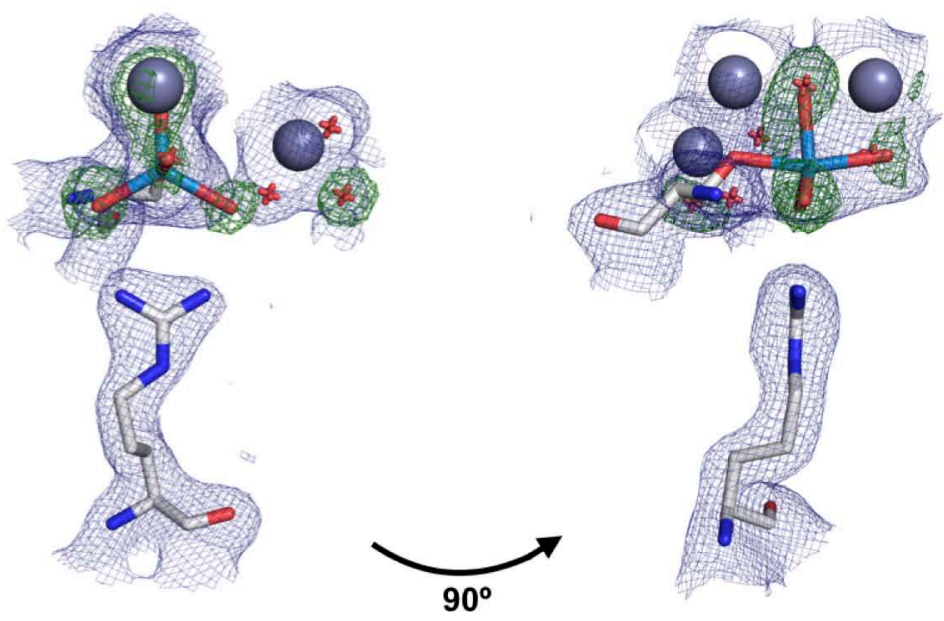

b
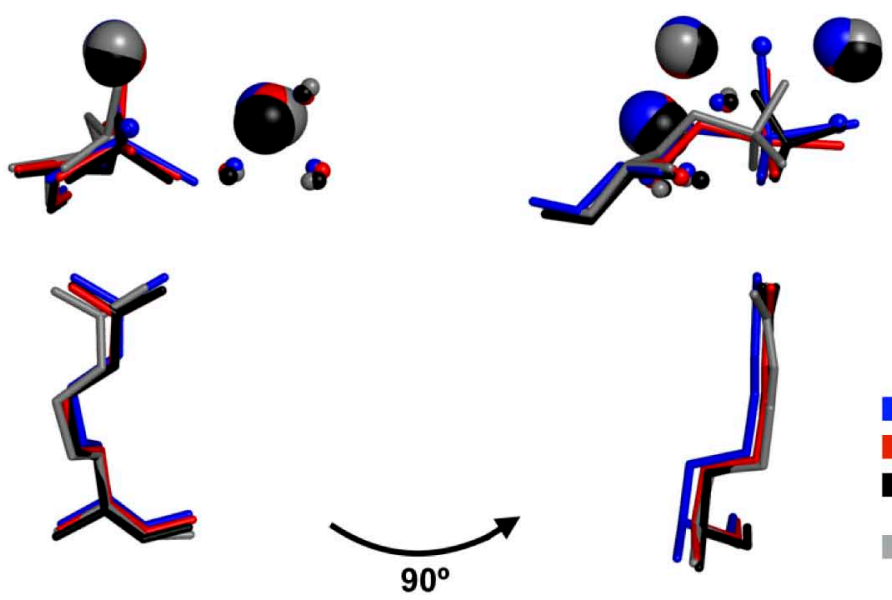

tungstate

vanadate

non-covalent

phosphate

covalent phosphate 
Figure 3 a

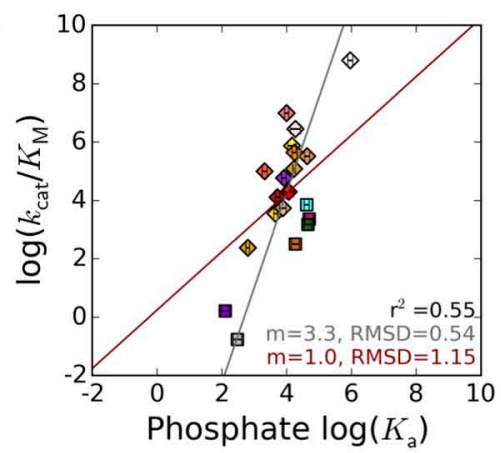

d

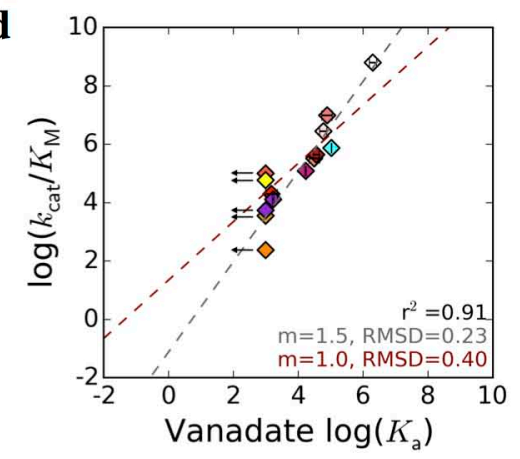

b

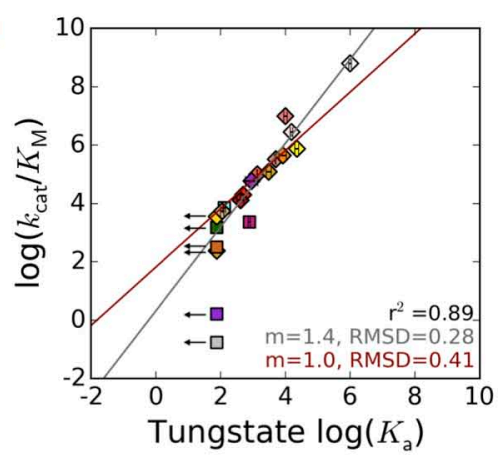

c

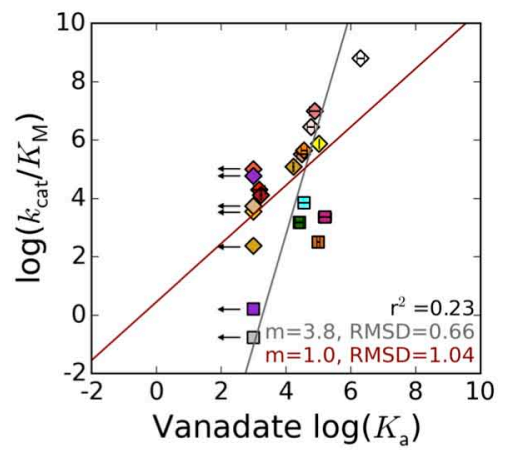

e

\begin{tabular}{|llll|}
\hline$\diamond$ & WT & $\diamond$ & D153A/K328A \\
$\diamond$ & D101A & $\square$ & D153A/E322Y \\
$\diamond$ & D153A & $\diamond$ & R166S/K328A \\
$\diamond$ & R166S & $\square$ & R166S/E322Y \\
$\diamond$ & D101A/D153A & $\square$ & E322Y/K328A \\
$\diamond$ & D101A/R166S/D153A & $\diamond$ & D101A/D153A/K328A \\
$\square$ & E322Y & $\diamond$ & D153A/R166S/K328A \\
$\diamond$ & K328A & $\square$ & D153A/E322Y/K328A \\
$\diamond$ & D101A/R166S & $\diamond$ & D101A/D153A/R166S/K328A \\
$\diamond$ & D153A/R166S & $\square$ & D101A/R166S/D153A/E322A/K328A \\
\hline
\end{tabular}


Table 1: X-ray crystallographic data collection and refinement statistics

\begin{tabular}{ll}
\hline Data Collection & \\
\hline Space group & $\mathrm{P}_{3} 22$ \\
Unit cell axes & $161.3,161.3,139.4$ \\
$a, b, c(\AA)$ & $90.0,90.0,120.0$ \\
$\alpha, \beta, \gamma\left({ }^{\circ}\right)$ & 0.9774 \\
Wavelength $(\AA)$ & $52.74-2.03(2.08-2.03)^{\mathrm{a}}$ \\
Resolution range $(\AA)$ & $0.059(0.276)^{\mathrm{b}}$ \\
$\mathrm{R}_{\text {merge }}$ & $0.028(0.129)$ \\
$\mathrm{R}_{\text {pim }}$ & $14.1(3.9)$ \\
$\langle\mathrm{I}\rangle /\langle\sigma \mathrm{I}\rangle$ & $93.6(93.4)[82.3(83.9)])^{\mathrm{c}}$ \\
Completeness $(\%)$ & $5.6(5.7)[2.8(3.2)]$ \\
Multiplicity & $0.823(0.964)$ \\
$C C_{1 / 2}$ & \\
\hline Refinement & $52.74-2.03$ \\
\hline Resolution range $(\AA)$ & $64069(6309)$ \\
No. of unique reflections & 6731 \\
No. of atoms & \\
Average $\mathrm{B}$ factors $\left(\AA^{2}\right)$ & 40.1 \\
protein & 33.4 \\
solvent & 34.4 \\
ligands & \\
RMSD from standard geometry & \\
Bond length $(\AA)$ & 0.02 \\
Bond angles $\left({ }^{\circ}\right)$ & 1.9 \\
Ramachandran statistics & \\
Favored regions $(\%)$ & 98.1 \\
Allowed regions $(\%)$ & 1.9 \\
Outliers $(\%)$ & 0 \\
PDB code & $5 \mathrm{C} 666$ \\
\hline a V & \\
\hline & \\
\hline
\end{tabular}

${ }^{a}$ Values in parenthesis are for the highest resolution shell.

${ }^{\mathrm{b}} \mathrm{R}_{\text {merge }}=\Sigma\left|\mathrm{I}_{\text {obs }}-\mathrm{I}_{\text {ave }}\right| / \Sigma \mathrm{I}_{\text {obs }}$

${ }^{\mathrm{c}}$ Values in brackets are for anomalous reflections. 
Table 2: Inhibition constants and catalytic efficiencies for AP mutants

\begin{tabular}{|c|c|c|c|c|}
\hline & Phosphate & $\begin{array}{c}K_{\mathrm{i}}^{\mathrm{a}}(\mu \mathrm{M}) \\
\text { Tungstate }\end{array}$ & Vanadate & $\begin{array}{r}k_{\text {cat }} / K_{\mathrm{M}} \mathrm{b} \\
\left(\mathrm{M}^{-1} \mathrm{~s}^{-1}\right)\end{array}$ \\
\hline WT & $1.1(0.2)^{\mathrm{c}}$ & $1.0(0.1)^{\mathrm{d}}$ & $0.50^{\mathrm{e}}$ & $6.3 \times 10^{8}$ \\
\hline D101A & $100(14)$ & $99(12)$ & $13(5)$ & $9.9 \times 10^{6}$ \\
\hline D153A & $53(19)$ & $64(6)$ & $17(0)$ & $2.8 \times 10^{6}$ \\
\hline R166S & $4.8(0.8) \times 10^{2 \mathrm{f}}$ & $7.4(0.1) \times 10^{2}$ & $\geq 1 \times 10^{3}$ & $1.0 \times 10^{5}$ \\
\hline D101A/D153A & $23(4)$ & $2.0(0) \times 10^{2}$ & $32(12)$ & $3.3 \times 10^{5}$ \\
\hline $\mathrm{D} 101 \mathrm{~A} / \mathrm{R} 166 \mathrm{~S} / \mathrm{D} 153 \mathrm{~A}$ & $85(30)$ & $2.0(0.4) \times 10^{3}$ & $6.7(2.8) \times 10^{2}$ & $2.0 \times 10^{4}$ \\
\hline E322Y & $24(4)$ & $7.7(0.8) \times 10^{3}$ & $28(12)$ & $7.2 \times 10^{3}$ \\
\hline K328A & $68(20)$ & $44(6)$ & $9.4(0.2)$ & $7.5 \times 10^{5}$ \\
\hline D101A/R166S & $1.2(0.2) \times 10^{2}$ & $1.1(0.5) \times 10^{3}$ & $\geq 1 \times 10^{3}$ & $5.8 \times 10^{4}$ \\
\hline D153A/R166S & $1.9(0.3) \times 10^{2}$ & $2.4(0.6) \times 10^{3}$ & $5.9(0 . \overline{3}) \times 10^{2}$ & $1.3 \times 10^{4}$ \\
\hline $\mathrm{D} 153 \mathrm{~A} / \mathrm{K} 328 \mathrm{~A}$ & $57(10)$ & $1.2(0.3) \times 10^{2}$ & $28(6)$ & $4.4 \times 10^{5}$ \\
\hline $\mathrm{D} 153 \mathrm{~A} / \mathrm{E} 322 \mathrm{Y}$ & $20(6)$ & $1.3(0.1) \times 10^{3}$ & $6.3(3.0)$ & $2.3 \times 10^{3}$ \\
\hline $\mathrm{D} 153 \mathrm{~A} / \mathrm{E} 322 \mathrm{~A}^{\mathrm{g}}$ & $12(5)$ & $1.8 \times 10^{3}$ & $47(0)$ & - \\
\hline R166S/K328A & $1.6(0.2) \times 10^{3}$ & $\geq 1.3 \times 10^{4}$ & $\geq 1 \times 10^{3}$ & $2.4 \times 10^{2}$ \\
\hline R166S/E322Y & $7.8(2.3) \times 10^{3}$ & $\geq 1.3 \times 10^{4}$ & $\geq 1 \times 10^{3}$ & 1.6 \\
\hline $\mathrm{E} 322 \mathrm{Y} / \mathrm{K} 328 \mathrm{~A}$ & $22(6)$ & $\geq 1.3 \times 10^{4}$ & $39(11)$ & $1.5 \times 10^{3}$ \\
\hline $\mathrm{D} 101 \mathrm{~A} / \mathrm{D} 153 \mathrm{~A} / \mathrm{K} 328 \mathrm{~A}$ & $58(5)$ & $3.3(0.6) \times 10^{2}$ & 59 (3) & $1.2 \times 10^{5}$ \\
\hline D153A/R166S/K328A & $2.2(0.3) \times 10^{2}$ & $\geq 1.3 \times 10^{4}$ & $\geq 1 \times 10^{3}$ & $3.6 \times 10^{3}$ \\
\hline $\mathrm{D} 153 \mathrm{~A} / \mathrm{E} 322 \mathrm{Y} / \mathrm{K} 328 \mathrm{~A}$ & $53(14)$ & $\geq 1.3 \times 10^{4}$ & $10(1)$ & $3.2 \times 10^{2}$ \\
\hline $\mathrm{D} 101 \mathrm{~A} / \mathrm{D} 153 \mathrm{~A} / \mathrm{R} 166 \mathrm{~S} / \mathrm{K} 328 \mathrm{~A}$ & $1.3(0.3) \times 10^{2}$ & $8.9(1.0) \times 10^{3}$ & $\geq 1 \times 10^{3}$ & $5.5 \times 10^{3}$ \\
\hline D101A/R166S/D153A/E322A/K328A & $3.3(1.0) \times 10^{3}$ & $\geq 1.3 \times 10^{4}$ & $\geq 1 \times 10^{3}$ & 0.17 \\
\hline
\end{tabular}

${ }^{\mathrm{a}}$ The listed values correspond to the observed, $\mathrm{pH}$-dependent $K_{\mathrm{i}}$, in contrast to the species-specific $K_{\mathrm{i}}$ listed in Fig. 1b. Errors are noted in parenthesis and correspond to the standard deviations from 2-3 independent experiments.

${ }^{\mathrm{b}}$ Values are reproduced from Sunden et al. [18], in which rates were measured by monitoring hydrolysis of $p$-nitrophenol phosphate (pNPP) for all AP variants except wild-type (WT), because the chemical step is not rate-limiting for WT AP with this substrate [3]. The chemical step is, however, rate-limiting for WT hydrolysis of the less reactive substrate methyl phosphate [3], so the WT $k_{\text {cat }} / K_{\mathrm{M}}$ value listed is the $k_{\text {cat }} / K_{\mathrm{M} \text {, obs }}$ value measured for methyl phosphate multiplied by the ratio of the $k_{\mathrm{cat}} / K_{\mathrm{M}}$ values observed for pNPP and methyl phosphate for AP mutants for which the chemical step is rate-limiting. This ratio of 20 is constant across several mutants, supporting its use as a correction factor to estimate the expected $k_{\text {cat }} / K_{\mathrm{M}}$ value [18].

' Value is reproduced from O'Brien et al. [3].

${ }^{\mathrm{d}}$ Value is averaged from O'Brien et al. [3] and Andrews et al. [6].

${ }^{\mathrm{e}}$ L. D. Andrews and D. Herschlag, unpublished result.

${ }^{\mathrm{f}} \mathrm{A}$ similar value of $4.6 \times 10^{2} \mu \mathrm{M}$ has been reported for this mutant [5].

${ }^{g}$ Inhibition constants for the D153A/E322A mutant were measured to test whether the differential responses of tungstate and vanadate to ablation of the $\mathrm{Mg}^{2+}$ ion arise from interactions with the Tyr introduced at this position. The D153A/E322Y and D153A/E322A mutants have similar inhibition constants for phosphate and tungstate, but vanadate binds 7.5-fold stronger when E322 is replaced with Tyr rather than Ala. A favorable interaction between vanadate and E322Y is the simplest model for this difference and would weaken the correlation between vanadate affinity and enzymatic activity. To assess the consequences of this effect, we adjusted vanadate inhibition constants for mutants containing E322Y by a factor of 7.5-fold, assuming a constant Tyr effect, and recalculated the linear fit. Even with this adjustment, the vanadate correlation is worse than that observed for tungstate, with a slope of $m=2.4$ (RMSD $=0.43$ ) for the total least squares regression linear fit and a coefficient of determination of $r^{2}=0.67$, whereas the corresponding values for the tungstate correlation are $\mathrm{m}=1.4(\mathrm{RMSD}=0.28)$ and $\mathrm{r}=0.89$. When the linear regression is re-calculated with the slope constrained to $m=1$, the RMSD of the fit for the vanadate data with this correction factor is 0.79 , compared to an RMSD of 0.41 for the tungstate data. 
Table 3: Transition state analog behavior for phosphate, tungstate, and vanadate.

\begin{tabular}{lccc}
\hline & Phosphate & Tungstate & Vanadate \\
\hline $\begin{array}{l}\text { Coefficient of } \\
\text { determination, } \mathrm{r}^{2}\end{array}$ & 0.55 & 0.89 & $0.23[0.91]^{\mathrm{a}}$ \\
$\mathrm{m}_{\text {best fit }}{ }^{\mathrm{b}}$ & 3.3 & 1.4 & $3.8[1.5]$ \\
RMSD $_{\text {best fit }}{ }^{\mathrm{c}}$ & 0.54 & 0.28 & $0.66[0.23]$ \\
RMSD $_{\mathrm{m}=1} \mathrm{~d}$ & 1.15 & 0.41 & $1.04[0.40]$ \\
\hline
\end{tabular}

${ }^{a}$ Brackets denote values calculated after excluding mutants with an ablated $\mathrm{Mg}^{2+}$ site.

${ }^{b}$ Slope of the best fit line was determined from orthogonal distance regression; see Methods.

${ }^{c}$ Root-mean-squared distance (RMSD) was calculated from the orthogonal residual between data points and the noted linear model.

${ }^{d}$ RMSD was calculated between data points and the best fit linear model with slope constrained to 1 . 


\section{Graphical Abstract}
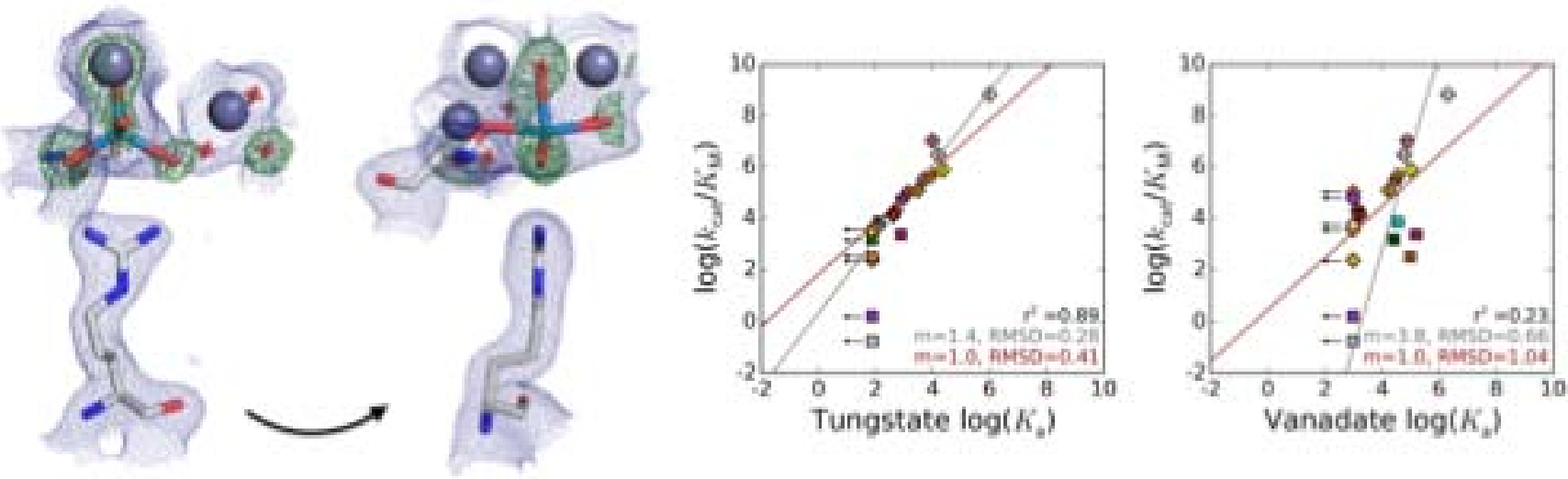